\title{
Exact solution of the zero-range process: fundamental diagram of the corresponding exclusion process
}

\author{
Masahiro Kanai \\ Graduate School of Mathematical Sciences, The University of Tokyo, 3-8-1 Komaba, \\ Tokyo 153-8914, Japan \\ E-mail: kanai@ms.u-tokyo.ac.jp
}

\begin{abstract}
In this paper, we propose a general way of computing expectation values in the zero-range process, using an exact form of the partition function. As an example, we provide the fundamental diagram (the flux-density plot) of the asymmetric exclusion process corresponding to the zero-range process. We express the partition function for the steady state by the Lauricella hypergeometric function, and thereby have two exact fundamental diagrams each for the parallel and random sequential update rules. Meanwhile, from the viewpoint of equilibrium statistical mechanics, we work within the canonical ensemble but the result obtained is certainly in agreement with previous works done in the grand canonical ensemble.
\end{abstract}

\section{Introduction}

The zero-range process (ZRP) is a stochastic model in which many indistinguishable particles occupy sites on a lattice [1, 2, 3]. Each site of the lattice may contain an integer number of particles and the particles hop to the next site with a probability which depends on the number of particles at the departure site. In other words, each particle interacts with the particles at the same site, i.e., they have a zero-range interaction. The hop probability is totally asymmetric, i.e., particles can only move in the definite direction and hence the flow of particles, even from the macroscopic viewpoint, never vanishes in general. The flow gives rise to a contrasting density distribution of particles, and one may therefore regard the ZRP as a typical nonequilibrium system [4, 5]. Since one can choose any function as the hop probability function, the ZRP has been extensively studied and applied for a wide variety of many-particle systems [6, 7, 8].

Although there now exist a lot of generalized/extended versions of the ZRP [3, 5, 9, in this paper we focus exclusively on the original one, i.e., the ZRP in one dimension and with periodic boundary. Updates of the particle configuration occur at each discrete time step, and two typical update rules, the parallel and random sequential update rules, are considered. These rules are defined as follows. Parallel update rule: at each time step, simultaneously at every site, one particle attempts to hop to the next site with its 
hop probability. Random sequential update rule: at each time step, a site is selected at random and then one particle at the site attempts to hop to the next site with its hop probability.

It is notable that the ZRP in one dimension can be mapped onto an exclusion process, i.e., a many-particle system on a periodic lattice whose sites contain a single particle or none [3]. Hereafter we call particles in the exclusion process vehicle to prevent confusion. According to this mapping, particles in the ZRP are regarded as the distance between adjacent vehicles in the exclusion process, i.e., the number of particles at site $i$ indicates the number of blank sites in front of vehicle $i$ following vehicle $i-1$; accordingly, for a particle to hop to the next site in the ZRP is for a vehicle to hop to the next site in the exclusion process. This exclusion process adequately simulates a traffic flow in which vehicles hop forward each with its own probability depending on the distance to the front vehicle. Note that the number of sites in the exclusion process is equal to the sum of sites and particles in the ZRP and meanwhile the number of vehicles is equal to that of sites in the ZRP.

\section{Nonequilibrium steady state and partition function}

In this section, following [3], we precisely define the nonequilibrium steady state of the ZRP and then formulate the partition function in that steady state. As well as equilibrium statistical mechanics, expectation values of physical quantities should be given by using that partition function.

\subsection{Nonequilibrium steady state}

Let $P\left(\left\{n_{m}\right\}\right)$ be the probability of finding the system in a configuration $\left\{n_{m}\right\}=$ $\left\{n_{1}, n_{2}, \ldots, n_{M}\right\}$, where $n_{m}$ denotes the number of particles at the $m$ th site. The transition probability from $\left\{n_{m}^{\prime}\right\}$ to $\left\{n_{m}\right\}$, denoted by $T\left(\left\{n_{m}\right\} \mid\left\{n_{m}^{\prime}\right\}\right)$, is expressed by the hop probability function $u(n)$ respectively according to the update rule, i.e., parallel and random sequential update rules. Note that it is always true that $u(0)=0$.

We firstly consider the parallel update rule. The transition probability for the update rule is given by

$$
T\left(\left\{n_{m}\right\} \mid\left\{n_{m}^{\prime}\right\}\right)=\sum_{\nu_{1}=0}^{1} \cdots \sum_{\nu_{M}=0}^{1}\left[\prod_{m=1}^{M} u\left(n_{m}^{\prime}\right) \delta\left(n_{m}-n_{m}^{\prime}+\nu_{m}-\nu_{m-1}\right)\right],
$$

where, at each site, $\nu_{m}(=0,1)$ particle hops to the next site with probability $u\left(n_{m}\right)$, and $\delta(n)$ is the Kronecker delta that returns unity if $n=0$ and zero otherwise. The balance of probability currents at each configuration $\left\{n_{m}\right\}$ is represented by the following equation:

$$
\sum_{\left\{n_{m}^{\prime}\right\}}\left[T\left(\left\{n_{m}\right\} \mid\left\{n_{m}^{\prime}\right\}\right) P\left(\left\{n_{m}^{\prime}\right\}\right)-T\left(\left\{n_{m}^{\prime}\right\} \mid\left\{n_{m}\right\}\right) P\left(\left\{n_{m}\right\}\right)\right]=0 .
$$


Then, we call a solution of (2) the nonequilibrium steady state probability (or the steady state probability, simply). The steady state probability $P\left(\left\{n_{m}\right\}\right)$ is given as a product of the single-site weights $f(n)$ :

$$
P\left(\left\{n_{m}\right\}\right)=\frac{1}{Z_{M, N}} \prod_{m=1}^{M} f\left(n_{m}\right) \quad\left(n_{1}+n_{2}+\cdots+n_{M}=N\right),
$$

where a normalization $Z_{M, N}$, the sum of the products over all configurations, is referred to as the partition function for the ZRP in the nonequilibrium steady state. One can directly confirm that (3) is a solution of (2); meanwhile the single-site weight is found to be

$$
f(n)= \begin{cases}1-u(1) & (n=0) \\ \frac{1-u(1)}{1-u(n)} \prod_{j=1}^{n} \frac{1-u(j)}{u(j)} & (n \geq 1) .\end{cases}
$$

Next, we consider the random sequential update rule. In this case, one can describe the steady state condition in a simple form:

$$
\sum_{m=1}^{M}\left[u\left(n_{m-1}+1\right) P\left(\ldots, n_{m-1}+1, n_{m}-1, \ldots\right)-u\left(n_{m}\right) P\left(\left\{n_{m}\right\}\right)\right] \theta\left(n_{m}\right),
$$

where $\theta\left(n_{m}\right)$ is the Heaviside function, which emphasizes that site $m$ must be occupied for there to be associated hops out of and into the configuration $\left\{n_{m}\right\}$. As well as in the case of the parallel update rule, the steady state probability $P\left(\left\{n_{m}\right\}\right)$ is given in the

product form (3), where the single-site weight for the random sequential update rule is

$$
f(n)= \begin{cases}1 & (n=0) \\ \prod_{j=1}^{n} \frac{1}{u(j)} & (n \geq 1) .\end{cases}
$$

(See [3] and [10] for details.)

\subsection{Partition function}

It should be noted that $f(n)$ is not identical to the probability that a given site (e.g. site 1 ) contains $n$ particles. Let the probability denoted by $p(n)$, and it is obtained from the probability distribution of configurations $P\left(\left\{n_{m}\right\}\right)$ as

$$
p(n)=\sum_{n_{2}+n_{3}+\cdots+n_{M}=N-n} P\left(\left\{n, n_{2}, \ldots, n_{M}\right\}\right)=f(n) \frac{Z_{M-1, N-n}}{Z_{M, N}} .
$$

The sum of $p(n)$ over $n$ is unity by definition, and we thereby obtain the recursion formula for the partition functions:

$$
\begin{aligned}
& Z_{M, N}=\sum_{n=0}^{N} f(n) Z_{M-1, N-n} \quad(M>1, N \geq 0), \\
& Z_{1, k}=f(k) \quad(k \geq 1) .
\end{aligned}
$$


Considering the generating functions, $\widehat{f}(\zeta):=\sum_{\widehat{Z}_{n=0}}^{\infty} f(n) \zeta^{n}$ and $\widehat{Z}_{M}(\zeta):=\sum_{n=0}^{\infty} Z_{M, n} \zeta^{n}$, we have a recursion with respect only to $M: \widehat{Z}_{M}(\zeta)=\widehat{f}(\zeta) \widehat{Z}_{M-1}(\zeta)$. Consequently, we find the fundamental relation between the two generating functions:

$$
\widehat{Z}_{M}(\zeta)=(\widehat{f}(\zeta))^{M}
$$

Thus, the partition function $Z_{M, N}$ is obtained from the single-site weight $f(n)$ via their generating functions. From the statistical mechanical viewpoint, $\widehat{Z}_{M}(\zeta)$ is appropriate for us to call the grand-canonical partition function, where we should think of $\zeta$ as the fugacity [2, 3].

Using $p(n)$ given in (7), the average velocity of vehicles in the steady state is defined by

$$
v_{M, N}=\sum_{n=0}^{N} u(n) p(n) .
$$

In the case of parallel update rule, we find that the average velocity is expressed by the partition function as

$$
v_{M, N}=-\frac{\sum_{n=0}^{N-1}(-1)^{n} Z_{M, n}}{(-1)^{N} Z_{M, N}} .
$$

In the case of random sequential update rule, we also find

$$
v_{M, N}=\frac{Z_{M, N-1}}{Z_{M, N}}
$$

(See [12] for details.)

\section{Exact solution}

In this section, we exactly compute the partition function and then the expectation value of the average velocity. These computations are fully done by using the formulae for the Lauricella hypergeometric function. The definition and formulae used are relegated to Appendix.

\subsection{Parallel update rule}

We start with the hop probability function $u(n)$ given as

$$
\begin{cases}u(0)=0, & \\ 0<u(n)<1 & (1 \leq \forall n \leq K), \\ u(n)=1 & (\forall n \geq K+1),\end{cases}
$$

where $K(\geq 1)$ is a constant integer. This is not a strong restriction, for one may take $K$ to be as large as necessary. As far as traffic-flow models are concerned, it is quite natural to choose this kind of hop probability function. 
Exact solution of the zero-range process

Substitution of (14) into (44) yields $f(n)=0$ for $n>K+1$, and we accordingly transform $\widehat{f}(\zeta)$ into a convenient form, i.e.,

$$
\widehat{f}(\zeta)=\sum_{n=0}^{K+1} f(n) \zeta^{n}=(1-u(1))(1+\zeta) \sum_{n=0}^{K}\left[\zeta^{n} \prod_{j=1}^{n} \frac{1-u(j)}{u(j)}\right],
$$

where we define $\prod_{j=1}^{n} a_{j}=1$ if $n=0$. For further computations, we formally factor the most right hand side of (15):

$$
\widehat{f}(\zeta)=f(0)(1+\zeta) \prod_{i=1}^{K}\left(1+x_{i} \zeta\right) .
$$

Then, comparing (15) with (16), we note the relation between $x_{1}, x_{2}, \ldots, x_{K}$ and $u(1), u(2), \ldots, u(K)$, namely

$$
e_{0}(x)=1, \quad e_{n}(x)=\prod_{j=1}^{n} \frac{1-u(j)}{u(j)} \quad(n=1,2, \ldots, K),
$$

where $e_{n}(x)$ is the $n$th elementary symmetric function of $K$ variables, $x=\left(x_{1}, \ldots, x_{K}\right)$. (Note that the elementary symmetric functions are defined by its generating function: $\prod_{i}\left(1+x_{i} \zeta\right)=\sum_{n} e_{n}(x) \zeta^{n}$, and specially $e_{0}(x)=1$.)

Accordingly, substitution of (16) into (10) yields

$$
\widehat{Z}_{M}(\zeta)=f(0)^{M}(1+\zeta)^{M} \prod_{i=1}^{K}\left(1+x_{i} \zeta\right)^{M}
$$

Compare (18) with (A.2), the generating function for the Lauricella hypergeometric function $F_{\mathrm{D}}$, and one finds an expression for the partition function in the nonequilibrium steady state: if $N \leq K M+M$ then

$$
Z_{M, N}=f(0)^{M}(-1)^{N} \frac{(-M)_{N}}{(1)_{N}} F_{\mathrm{D}}(-N, \overbrace{-M, \ldots,-M}^{K}, M-N+1 ; x),(1
$$

and otherwise $Z_{M, N}=0$. (See Appendix A for definition of the notation.) As seen below, this expression using the Lauricella hypergeometric function is quite useful for exact computation of expectation values.

In the case that $N>K M+M$ the average velocity in the steady state takes the value of unity, since all vehicles take more than $K$-site distance from the front vehicle (or all sites in the corresponding ZRP contain more than $K$ particles). If $N \leq K M+M$, by substituting of (19) into (12) we have an expression for the average velocity. Using a recursion formula for the Lauricella hypergeometric function with respect to parameters Appendix A.2,

$$
\alpha F_{\mathrm{D}}(\alpha+1, \beta, \gamma+1 ; x)-\gamma F_{\mathrm{D}}(\alpha, \beta, \gamma ; x)=(\alpha-\gamma) F_{\mathrm{D}}(\alpha, \beta, \gamma+1 ; x),
$$

the sum in the numerator in (12) is firstly carried out:

$$
\begin{aligned}
\sum_{n=0}^{N-1} \frac{(-M)_{n}}{(1)_{n}} & F_{\mathrm{D}}(-n,-M, \ldots,-M, M-n+1 ; x) \\
& =\frac{(1-M)_{N-1}}{(1)_{N-1}} F_{\mathrm{D}}(1-N,-M, \ldots,-M, M-N+1 ; x) .
\end{aligned}
$$


Accordingly, the average velocity of vehicles for arbitrary numbers of sites and vehicles is given as

$$
v_{M, N}=\frac{N}{M} \frac{F_{\mathrm{D}}(1-N,-M, \ldots,-M, M-N+1 ; x)}{F_{\mathrm{D}}(-N,-M, \ldots,-M, M-N+1 ; x)} .
$$

We remark that (22) is valid for arbitrary numbers of $M$ and $N$.

Then, we expand (22) in lattice size $L=M+N$ with a fixed density of vehicles $\rho=M / L$. Our strategy to take the thermodynamic limit is to eliminate the hypergeometric function from (22). The Lauricella hypergeometric differential equation that $Y=F_{\mathrm{D}}(\alpha, \beta, \gamma ; x)$ satisfies is defined by

$$
\left[(\gamma-1+\delta) \delta_{i}-x_{i}(\alpha+\delta)\left(\beta_{i}+\delta_{i}\right)\right] Y=0 \quad(i=1,2, \ldots, K),
$$

where $\delta=\sum_{i} \delta_{i}$ and $\delta_{i}=x_{i} \partial / \partial x_{i}$. Using a differentiation formula (A.11), we have

$$
F_{\mathrm{D}}(1-N,-M, \ldots,-M, M-N+1 ; x)=y-\frac{1}{N} \delta y,
$$

where $y=F_{\mathrm{D}}(-N,-M, \ldots,-M, M-N+1 ; x)$, the denominator in (22), and then substitution of this into (22) yields

$$
\delta y=\frac{N}{h}\left(h-v_{M, N}\right) y \quad\left(h=\frac{N}{M}\right)
$$

Meanwhile, we consider the hypergeometric differential equation for $y$ (where $\alpha=-N$, $\beta_{i}=-M$ and $\left.\gamma=M-N+1\right)$ and find

$$
\delta_{i} y=\frac{N}{h} \frac{v_{M, N} x_{i}+\frac{h}{N}\left(1-x_{i}\right) \delta_{i} v_{M, N}}{1-v_{M, N}+v_{M, N} x_{i}} y \quad(i=1,2, \ldots, K) .
$$

Since $\sum_{i} \delta_{i} y=\delta y$, equating the sum of the right hand side of (26) with that of (25) we successfully eliminate $y$ :

$$
h-v_{M, N}=\sum_{i=1}^{K} \frac{v_{M, N} x_{i}+\frac{h}{N}\left(1-x_{i}\right) \delta_{i} v_{M, N}}{1-v_{M, N}+v_{M, N} x_{i}} .
$$

Expand the average velocity as $v_{M, N}=v_{0}+v_{1} L^{-1}+v_{2} L^{-2}+\cdots$, and substitution of this into (26) yields a series of equations for $v_{0}, v_{1}, v_{2}, \ldots$. Consequently, as $N$ tends to infinity, we have

$$
h-v=\sum_{i=1}^{K} \frac{v x_{i}}{1-v+v x_{i}},
$$

where $v\left(=v_{0}\right)$ is the average velocity in the thermodynamic limit. Solving the series of equations for $v_{1}, v_{2}, \ldots$ in turn, one can obtain the higher-order correction terms as functions of $v$. The computations are straightforward, but however the expressions of those terms are too long to be included.

In [10], they already obtained the same result as (28) working in the grand-canonical ensemble where the fluctuation of the particle number $N$ is allowed. Actually, (28) is consistent with their result,

$$
\langle N\rangle=w \frac{\partial}{\partial w} \log \widehat{Z}_{M}(w),
$$


where $\langle N\rangle$ means the expectation value of the particle number in the grand-canonical ensemble. Since the right hand side of (28) is in a symmetric form with respect to $x_{1}, \ldots, x_{K}$, one can express it using the elementary symmetric functions of them. Recall $\prod_{i}\left(1+x_{i} \zeta\right)=\sum_{n} e_{n}(x) \zeta^{n}$, and one finds the identity,

$$
\sum_{i=1}^{K} \frac{w x_{i}}{1+w x_{i}}=\frac{\sum_{r=1}^{K} r e_{r}(x) w^{r}}{\sum_{r=0}^{K} e_{r}(x) w^{r}} .
$$

The left hand side of (30) is equivalent to the right hand side of (28) if one lets $w=v /(1-v)$, and thus we find that (28) is equivalent to (29).

We note that using (29) one has the fundamental diagram in a parametric representation: flux $Q(v)=\rho(v) v$ and density $\rho(v)=1 /(1+h)$, where $v(0 \leq v \leq 1)$ is the parameter. As an example, we illustrate a fundamental diagram choosing a hop probability function that approximately describes motion of vehicles in traffic flow, i.e.,

$$
u(n)=\frac{\tanh (n-c)+\tanh c}{1+\tanh c},
$$

where we let $c=3 / 2$. Note that the hop probability function can be estimated from real traffic data, and (31) is often chosen for traffic-flow models [11, 13]. Figure 11 shows the exact fundamental diagram illustrated by using (29) with $K=50$, and the simulation data in addition. They show complete agreement.

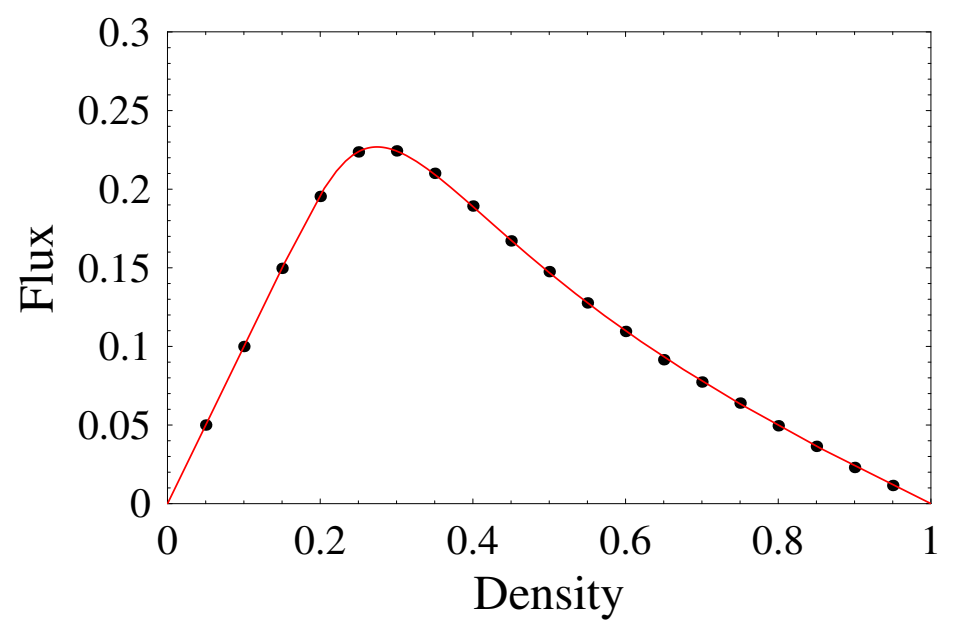

Figure 1. A fundamental diagram of the exclusion process corresponding to the zerorange process with the parallel update rule. Gray line shows the exact solution given in the parametric representation, and black points are simulation data. Numerical simulation is done with site $L=1000$. These are in good agreement.

\subsection{Random sequential update rule}

In the same way as in the case of the parallel update rule, we obtain the fundamental diagram for the ZRP with the random sequential update rule. 
The single-site weight is given in (6), and then the generating function for these weights is given as

$$
\widehat{f}(\zeta)=\sum_{n=0}^{\infty}\left[\zeta^{n} \prod_{j=1}^{n} \frac{1}{u(j)}\right]
$$

where $u(n)$ is given in (14). Then, we can factor (32) into $\widehat{f}(\zeta)=(1-\zeta)^{-1} \prod_{i=1}^{K}\left(1-x_{i} \zeta\right)$ and express the partition function by the Lauricella hypergeometric function:

$$
Z_{M, N}=\frac{(M)_{N}}{(1)_{N}} F_{\mathrm{D}}(-N, \overbrace{-M, \ldots,-M}^{K},-M-N+1 ; x),
$$

where the independent variables $x=\left(x_{1}, x_{2}, \ldots, x_{K}\right)$ satisfy

$$
e_{0}(x)=1, \quad e_{n}(x)=\prod_{j=1}^{n} \frac{1}{u(j)} \quad(n=1,2, \ldots, K) .
$$

From (13) and (33), the average velocity for the finite numbers, $M$ and $N$, is expressed by the Lauricella hypergeometric function:

$$
v_{M, N}=\frac{N}{M+N-1} \frac{F_{\mathrm{D}}(-N+1,-M, \ldots,-M,-M-N+2 ; x)}{F_{\mathrm{D}}(-N,-M, \ldots,-M,-M-N+1 ; x)} .
$$

Thus, applying the formulae for the hypergeometric functions we find

$$
h=\frac{N}{M}=\left(1-\frac{1}{M}\right) \frac{v_{M, N}}{1-v_{M, N}}-\sum_{i=1}^{K} \frac{x_{i} v_{M, N}+\frac{1-x_{i}}{\left(1-v_{M, N}\right) M} \delta_{i} v_{M, N}}{1-x_{i} v_{M, N}} .
$$

As well as the parallel update case, we can expand $v_{M, N}$ as a series $v_{0}+v_{1} L^{-1}+v_{2} L^{-2}+\cdots$.

Especially in the thermodynamic limit, we recover the result given in $[6,3]$

$$
\langle N\rangle=v \frac{\partial}{\partial v} \log \widehat{Z}(v)
$$

which was obtained within the grand-canonical ensemble.

In contrast with the parallel-update case, if $K$ tends to infinity, we need to determine the range of parameter $v$. For this purpose, we impose a simple condition on the hop function, i.e.,

$$
\lim _{n \rightarrow \infty} u(n)=u_{\infty} \quad\left(0<u_{\infty} \leq 1\right),
$$

which surely allows us to determine the parameter range. Then, the range of values of $v$ becomes $0 \leq v<u_{\infty}$. It depends on the asymptotic behaviour of $u(n)$ whether $\widehat{f}(v)$ converges or not at $v=u_{\infty}$. The convergence of $\widehat{f}(v)$ is discussed in the context of condensation occurring in the ZRP [3, 14, 15].

We choose a traffic-flow model with the hop probability function (31) and random sequential update rule, and show the exact fundamental diagram and simulation result in figure 2. These are in good agreement as well as in the case of the parallel update rule.

We consider a simple hop probability function which allows us to provide a direct formula of fundamental diagram instead of the parametric representation, i.e.,

$$
u(1)=\lambda, \quad u(n)=p \quad(n \geq 2) .
$$




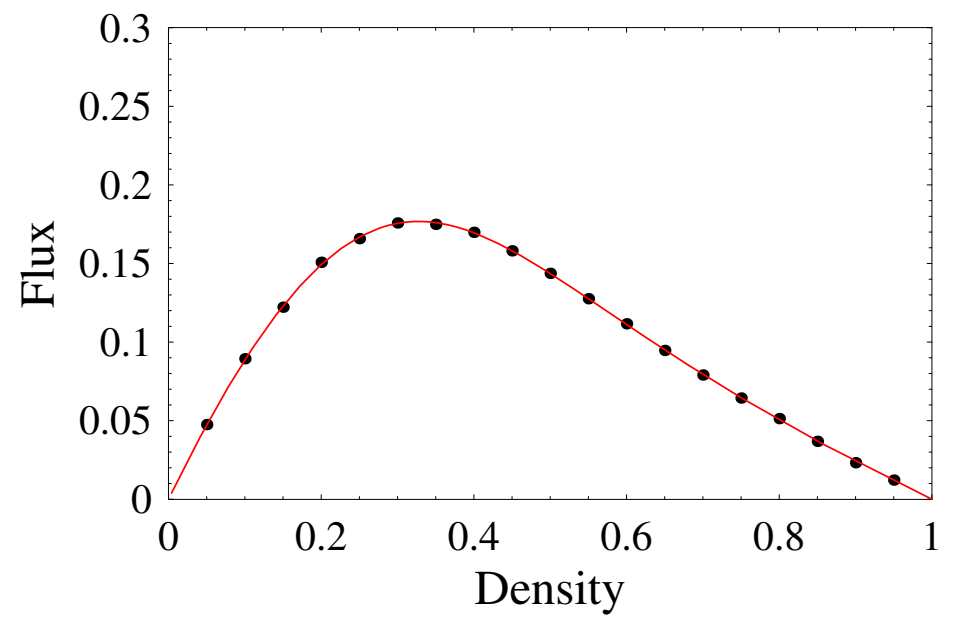

Figure 2. A fundamental diagram of the exclusion process corresponding to the zerorange process with the random sequential update rule. (See Fig.1 for comparison.) Gray line shows the exact solution given in the parametric representation, and black points are simulation data. Numerical simulation is done with site $L=1000$. These are in good agreement.

Substitution of (39) into (37) via (32) yields

$$
h=\frac{p^{2} v}{(p-v)(\lambda p+(p-\lambda) v)} .
$$

Accordingly, eliminating $v$ we obtain the fundamental diagram explicitly described by

$$
Q=p \rho\left[1-\frac{1-\sqrt{1-4(1-q) \rho(1-\rho)}}{2(1-q)(1-\rho)}\right] \text { where } q=\frac{\lambda}{p} .
$$

One can also figure out an explicit formula for this model in the case of the parallel update rule; however, one has to solve a cubic equation.

If we take the limit as $\lambda \rightarrow p$ (i.e., $q \rightarrow 1$ ) in (41), we recover the fundamental diagram of the ASEP, $Q=p \rho(1-\rho)$, which is a well-known solution [16]. If we let $p=1$ ( $q=\lambda$, accordingly), then we recover another exact solution given in [17], where they obtained the same result by using the matrix-product ansatz.

\section{Summary and remark}

In this paper, we provide an exact solution of the zero-range process: we have the partition function for the general case, which is expressed by the Lauricella hypergeometric function, and which allows us to make further computations. Although the solution obtained is rather formal, that is enough for us to compute expectation values of macroscopic quantities. In particular, using the exact partition function we obtain the average velocity of vehicles for arbitrary numbers of sites and particles. Moreover, expanding the average velocity in terms of the site number, we obtain the 
fundamental diagram as the leading term of the expansion. One can also figure out the correction terms through a long computation.

Due to the fact that the steady state of the ZRP has a factorized form, one can do within equilibrium statistical mechanics while dealing with the macroscopic quantities averaged over all the lattice. In this regard, one may take $Z_{M, N}$ as the canonical partition function in which the particle number is conserved. As a result, we compute the expectation value of the average velocity within the canonical ensemble. In general, statistical treatment in the canonical ensemble is more difficult than that in the grand canonical ensemble. Both results in these ensembles are equivalent in the thermodynamic limit. An analytical approach based on the grand canonical ensemble was previously made to the ZRP with the random sequential update rule in [6], and to the ZRP with the parallel update rule in [10]. Recently, canonical analysis of the condensation is also done [14, 15]. In this regard, it will be interesting to exactly compute the probability $p(n)$ that a single site is to contain $n$ particles in the steady state.

Recently, as a traffic-flow model, we introduce the stochastic optimal velocity (SOV) model [11, 18] in the form of combining two exactly solvable models, i.e., the asymmetric simple exclusion process (ASEP) [16] appearing as a special case of the ZRP, and the ZRP itself; however it does not seem to us that the model has an exact solution in the general case. In numerical simulations, we find that the SOV model has an intriguing and complex phase transition in the fundamental diagram, and our future goal is to analyse in detail the phase transition observed, e.g., by using a theoretical method for exactly solvable models.

\section{Acknowledgments}

The author thanks Teruhisa Tsuda for a helpful discussion on practical computations.

This work is supported by a grant for the 21st Century COE program at The University of Tokyo from the Ministry of Education, Culture, Sports, Science and Technology, Japan.

\section{Appendix A. The Lauricella hypergeometric function}

There are four types of the Lauricella hypergeometric function, being denoted by $F_{\mathrm{A}}$, $F_{\mathrm{B}}, F_{\mathrm{C}}$ and $F_{\mathrm{D}}$. Each of them has a series representation, an integral representation and a differential equation it satisfies. Since only $F_{\mathrm{D}}$ appears in this paper, we confine our attention to $F_{\mathrm{D}}$. (See [19] for details.)

Firstly, we define the Lauricella hypergeometric function $F_{\mathrm{D}}$ with $K$ arguments by

$$
F_{\mathrm{D}}\left(\alpha, \beta_{1}, \ldots, \beta_{K}, \gamma ; x_{1}, \ldots, x_{K}\right)=\sum_{m \in I_{K}} \frac{(\alpha)_{|m|}(\beta)_{m}}{(\gamma)_{|m|}(1)_{m}} x^{m},
$$

where $\alpha, \beta_{1}, \ldots, \beta_{K}$ and $\gamma$ are all complex parameters, $(a)_{n}=a(a+1) \cdots(a+n-1)$ is the Pochhammer symbol, and notations in the right hand side are defined as follows: $I_{K}=\left\{m=\left(m_{1}, \ldots, m_{K}\right) ; m_{i} \in \mathbb{Z}_{\geq 0}(i=1,2, \ldots, K)\right\}$, then, for $m \in I_{K}$, 
Exact solution of the zero-range process

$|m|=m_{1}+\cdots+m_{K},(\beta)_{m}=\left(\beta_{1}\right)_{m_{1}}\left(\beta_{2}\right)_{m_{2}} \cdots\left(\beta_{K}\right)_{m_{K}},(1)_{m}=(1)_{m_{1}}(1)_{m_{2}} \cdots(1)_{m_{K}}$ and $x^{m}=x_{1}{ }^{m_{1}} \cdots x_{K}{ }^{m_{K}}$. We also use the following notations for simplicity: $\beta=$ $\left(\beta_{1}, \beta_{2}, \ldots, \beta_{K}\right)$ and $x=\left(x_{1}, x_{2}, \ldots, x_{K}\right)$.

The hypergeometric differential equation for $F_{\mathrm{D}}(\alpha, \beta, \gamma ; x)$ is given in (23). We omit the integral representation because it is not used in the present work.

\section{Appendix A.1. Generating function}

We present the generating function for $F_{\mathrm{D}}$ :

$$
(1-\zeta)^{\kappa} \prod_{i=1}^{K}\left(1-x_{i} \zeta\right)^{-\beta_{i}}=\sum_{n=0}^{\infty} \frac{(-\kappa)_{n}}{(1)_{n}} F_{\mathrm{D}}(-n, \beta, \kappa-n+1 ; x) \zeta^{n} .
$$

Here, we give a proof of (A.2) by mathematical induction. One should note the relations,

$$
(1-z)^{a}=\sum_{n=0}^{\infty} \frac{(-a)_{n}}{(1)_{n}} z^{n}
$$

and

$$
(a)_{l-m}=\frac{a(a+1) \cdots(a+l-1)}{(a+l-m) \cdots(a+l-1)}=\frac{(-1)^{m}(a)_{l}}{(1-a-l)_{m}} .
$$

(i) If $K=1$, then one has

$$
\begin{aligned}
(1-\zeta)^{\kappa}\left(1-x_{1} \zeta\right)^{-\beta_{1}} & =\sum_{m_{1}=0}^{\infty} \sum_{k=0}^{\infty} \frac{(-\kappa)_{k}\left(\beta_{1}\right)_{m}}{(1)_{k}(1)_{m_{1}}} x_{1}{ }^{m_{1}} \zeta^{k+m_{1}} \\
& =\sum_{m_{1}=0}^{\infty} \sum_{n=m_{1}}^{\infty} \frac{(-\kappa)_{n-m_{1}}\left(\beta_{1}\right)_{m_{1}}}{(1)_{n-m_{1}}(1)_{m_{1}}} x_{1}^{m_{1}} \zeta^{n} \\
& =\sum_{n=0}^{\infty} \frac{(-\kappa)_{n}}{(1)_{n}} F_{\mathrm{D}}\left(-n, \beta_{1}, \kappa-n+1, x_{1}\right) \zeta^{n} .
\end{aligned}
$$

Thus, (A.2) holds if $K=1$.

(ii) Assume that (A.2) holds if $K=l-1$, and one accordingly has

$$
\begin{aligned}
(1-\zeta)^{\kappa} \prod_{i=1}^{l}\left(1-x_{i} \zeta\right)^{-\beta_{i}} & =\left(1-x_{l} \zeta\right)^{-\beta_{l}} \sum_{n=0}^{\infty} \frac{(-\kappa)_{n}}{(1)_{n}} F_{\mathrm{D}}\left(-n, \beta^{\prime}, \kappa-n+1 ; x^{\prime}\right) \zeta^{n} \\
& =\sum_{k=0}^{\infty} \sum_{n=0}^{\infty} \frac{(-\kappa)_{n}}{(1)_{n}} \zeta^{k+n} \sum_{m \in I_{l-1}} \frac{(-n)_{|m|}\left(\beta^{\prime}\right)_{m}\left(\beta_{l}\right)_{k}\left(x^{\prime}\right)^{m} x_{l}{ }^{k}}{(\kappa-n+1)_{|m|}(1)_{m}(1)_{k}} \\
& =\sum_{n=0}^{\infty} \frac{(-\kappa)_{n}}{(1)_{n}} \zeta^{n} \sum_{(m, k) \in I_{l}} \frac{(-n)_{|m|+k}\left(\beta^{\prime}\right)_{m}\left(\beta_{l}\right)_{k}\left(x^{\prime}\right)^{m} x_{l}{ }^{k}}{(\kappa-n+1)_{|m|+k}(1)_{m}(1)_{k}},
\end{aligned}
$$

where $x^{\prime}=\left(x_{1}, \ldots, x_{l-1}\right), \beta^{\prime}=\left(\beta_{1}, \ldots, \beta_{l-1}\right)$ and $I_{k}=\left\{m=\left(m_{1}, \ldots, m_{k}\right) ; m_{i} \in\right.$ $\left.\mathbb{Z}_{\geq 0}(i=1,2, \ldots, k)\right\}$.

Thus, (A.2) holds also when $K=l$. Therefore, we may conclude that (A.2) holds for any natural number $K$. 
Appendix A.2. Recursion formula

We provide a recursion with respect to the parameters, $\alpha$ and $\gamma$, for $F_{\mathrm{D}}$ :

$$
\alpha F_{\mathrm{D}}(\alpha+1, \beta, \gamma+1 ; x)=\gamma F_{\mathrm{D}}(\alpha, \beta, \gamma ; x)+(\alpha-\gamma) F_{\mathrm{D}}(\alpha, \beta, \gamma+1 ; x) .
$$

One should note simple formulae for the Pochhammer symbol,

$$
(a+1)_{n}-(a)_{n}=\frac{n}{a}(a)_{n}, \quad \text { and } \quad \frac{1}{(a)_{n}}-\frac{1}{(a+1)_{n}}=\frac{n}{(a)_{n+1}} .
$$

From (A.1) and the two formulae above, one respectively has

$$
F_{\mathrm{D}}(\alpha+1, \beta, \gamma ; x)-F_{\mathrm{D}}(\alpha, \beta, \gamma ; x)=\sum_{m \in I} \frac{|m|}{\alpha} \frac{(\alpha)_{|m|}(\beta)_{m}}{(\gamma)_{|m|}(1)_{m}} x^{m},
$$

and

$$
F_{\mathrm{D}}(\alpha, \beta, \gamma ; x)-F_{\mathrm{D}}(\alpha, \beta, \gamma+1 ; x)=\sum_{m \in I} \frac{|m|}{\gamma} \frac{(\alpha)_{|m|}(\beta)_{m}}{(\gamma+1)_{|m|}(1)_{m}} x^{m} \text {. }
$$

Then, we can construct (A.7) from (A.9) and (A.10).

\section{Appendix A.3. Differentiation formula}

Since the Lauricella hypergeometric function is a multivariable function, when taking the derivative one needs to deal with partial differentiation: $\delta_{i}=x_{i} \partial / \partial x_{i}$ and $\delta=\sum_{i} \delta_{i}$.

In the present work, we specially use the following formulae:

$$
\begin{aligned}
\delta F_{\mathrm{D}}(\alpha, \beta, \gamma ; x) & =\alpha\left(F_{\mathrm{D}}(\alpha+1, \beta, \gamma ; x)-F_{\mathrm{D}}(\alpha, \beta, \gamma ; x)\right) \\
& =(\gamma-1)\left(F_{\mathrm{D}}(\alpha, \beta, \gamma-1 ; x)-F_{\mathrm{D}}(\alpha, \beta, \gamma ; x)\right) .
\end{aligned}
$$

Note that $\delta_{i} x_{i}{ }^{m_{i}}=m_{i} x_{i}{ }^{m_{i}}$, and from (A.8) one finds

$$
\begin{aligned}
\delta F_{\mathrm{D}}(\alpha, \beta, \gamma ; x) & =\sum_{m \in I} \frac{(\alpha)_{|m|}(\beta)_{m}}{(\gamma)_{|m|}(1)_{m}}|m| x^{m} \\
& =\sum_{m \in I} \frac{\alpha\left((\alpha+1)_{|m|}-(\alpha)_{|m|}\right)(\beta)_{m}}{(\gamma)_{|m|}(1)_{m}} x^{m} \\
& =\sum_{m \in I}\left(\frac{\gamma-1}{(\gamma-1)_{|m|}}-\frac{\gamma-1}{(\gamma)_{|m|}}\right) \frac{(\alpha)_{|m|}(\beta)_{m}}{(1)_{m}} x^{m} .
\end{aligned}
$$

Thus, (A.11) is proven.

\section{References}

[1] Spitzer F 1970 Advances in Math. 5246

[2] Evans M R 2000 Braz. J. Phys. 3042

[3] Evans M R and Hanney T 2005 J. Phys. A: Math. Gen. 38 R195

[4] Schmittmann B and Zia R P K 1995 Phase Transitions and Critical Phenomena vol 17 (London: Academic Press)

[5] Schütz G M 2003 J. Phys. A: Math. Gen. 36 R339

[6] Bialas P, Burda Z and Johnston D 1997 Nuclear Physics B 493505 
[7] O'Loan O J, Evans M R and Cates M E 1998 Phys. Rev. E 581404

[8] Kunwar A, John A, Nishinari K, Schadschneider A and Chowdhury D 2004 J. Phys. Soc. Jpn. 73 2979

[9] Levine E, Mukamel D and Schütz G M 2005 J. Stat. Phys. 120759

[10] Evans M R 1997 J. Phys. A: Math. Gen. 305669

[11] Kanai M, Nishinari K and Tokihiro T 2005 Phys. Rev. E 72 035102(R)

[12] Kanai M, Nishinari K and Tokihiro T 2006 J. Phys. A: Math. Gen. 399071

[13] Bando M, Hasebe K, Nakayama A, Shibata A and Sugiyama Y 1995 Phys. Rev. E 511035 Bando M, Hasebe K, Nakanishi K, Nakayama A, Shibata A and Sugiyama Y 1995 J. Phys. I France 51389

Igarashi Y, Itoh K and Nakanishi K 1999 J. Phys. Soc. Jpn. 68791

Hasebe K, Nakayama A and Sugiyama Y 1999 Phys. Lett. A 259135

[14] Majumdar S N, Evans M R and Zia R K P 2005 Phys. Rev. Lett. 94180601

[15] Evans M R, Majumdar S N and Zia R K P 2006 J. Stat. Phys. 123357

[16] Rajewsky N, Santen L, Schadschneider A and Schreckenberg M 1998 J. Stat. Phys. 92151

[17] Klauck K and Schadschneider A 1999 Physica A 271102

[18] Kanai M, Nishinari K and Tokihiro T 2006 J. Phys. A: Math. Gen. 392921

[19] Erdélyi A 1953-55 Higher Transcendental Functions (MacGraw-Hill) 